\title{
Electrocardiography in people living at high altitude of Nepal
}

\author{
Nirmal Aryal, ${ }^{1}$ Mark Weatherall, ${ }^{1}$ Yadav Kumar Deo Bhatta, ${ }^{2}$ Stewart Mann ${ }^{1}$
}

'Department of Medicine, University of Otago, Wellington, New Zealand ${ }^{2}$ Norvic International Hospital, Kathmandu, Nepal

\section{Correspondence to}

Nirmal Aryal, Department of Medicine, University of Otago, Wellington, New Zealand, P. 0. Box 7343, Wellington 6021, New Zealand; nirmal.aryal@otago.ac.nz

Received 18 September 2016 Revised 4 January 2017 Accepted 9 January 2017

\section{CrossMark}

To cite: Aryal N, Weatherall $M$, Bhatta YKD, et al. Heart Asia 2017:9:48-53.

doi:10.1136/heartasia-2016010838

\section{ABSTRACT}

Objective The main objective of this study was to estimate the prevalence of coronary heart disease (CHD) of high-altitude populations in Nepal determined by an ECG recordings and a medical history.

Methods We carried out a cross-sectional survey of cardiovascular disease and risk factors among people living at four different altitude levels, all above $2800 \mathrm{~m}$, in the Mustang and Humla districts of Nepal. 12-lead ECGs were recorded on 485 participants. ECG recordings were categorised as definitely abnormal, borderline or normal.

Results No participant had Q waves to suggest past Qwave infarction. Overall, $5.6 \%(95 \% \mathrm{Cl} 3.7$ to 8.0$)$ of participants gave a self-report of CHD. The prevalence of abnormal (or borderline abnormal) ECG was 19.6\% ( $95 \% \mathrm{Cl} 16.1$ to 23.4). The main abnormalities were: right axis deviation in $5.4 \%(95 \% \mathrm{Cl} 3.5$ to 7.7$)$ and left ventricular hypertrophy by voltage criteria in 3.5\% (95\% Cl 2.0 to 5.5). ECG abnormalities were mainly on the left side of the heart for Mustang participants (Tibetan origin) and on the right side for Humla participants (Indo-Aryans). There was a moderate association between the probability of abnormal (or borderline abnormal) ECG and altitude when adjusted for potential confounding variables in a multivariate logistic model; with an OR for association per $1000 \mathrm{~m}$ elevation of altitude of $2.83(95 \% \mathrm{Cl} 1.07$ to 7.45$)$, $\mathrm{p}=0.03$.

Conclusions Electrocardiographic evidence suggests that although high-altitude populations do not have a high prevalence of CHD, abnormal ECG findings increase by altitude and risk pattern varies by ethnicity.

\section{INTRODUCTION}

More than 140 million people in the world, comprising $2 \%$ of the global population, live permanently at high altitude (HA), defined as at $>2500 \mathrm{~m}^{1}$ The Himalayan mountains of Asia, Andean mountains of South America and Ethiopian summits of northeast Africa are the major geographical locations of HA populations. ${ }^{1}$

The positive and negative effects induced by living at HA, and chronic hypobaric hypoxia, on cardiovascular health, are uncertain. Increased angiogenesis and structural vascular changes caused by HA may reduce blood pressure (BP) and promote cardiometabolic efficiency. However, erythrocytosis (from chronically low inspired oxygen concentrations) and pulmonary hypertension (HT) among those living at HA may be risk factors for adverse cardiovascular events. ${ }^{2}$ The largest HA populations, Tibetans and Andeans, exhibit different patterns of adaptation and physiological responses to environmental hypoxia. ${ }^{3}$ Past research shows that genetic factors account for some variance in levels of physiological adaptation of HA populations. ${ }^{4}$ These factors, in association with different lifestyle practices of HA populations, may contribute to research findings that the different HA populations have different risk for cardiovascular events. Despite more than five decades of research on cardiovascular health at $\mathrm{HA}$, the impact of hypoxia on cardiovascular health is still uncertain.

In Nepal, nearly 2 million people permanently live in mountain areas, representing $7 \%$ of the national population. ${ }^{5}$ Those with Tibetan ancestry are the dominant ethnic HA population in Nepal, for example Sherpa, Thakali, Tibetan Gurung, Lama.

There may be a relationship between usual resident altitude and cardiovascular health in Nepalese HA populations because of hypoxia-induced physiological responses. In addition, Tibetan-related populations are known to have a high prevalence of HT, which may raise their risk of coronary heart disease (CHD). There has been no research published on prevalence of CHD among HA populations in Nepal. The main purpose of this study was to investigate the prevalence of $\mathrm{CHD}$, determined by a medical history and an ECG recording, of people living at different levels of HA in Nepal.

\section{METHODS}

This was a cross-sectional study.

\section{Study sample}

Sixteen, out of 75 districts of Nepal, are classified as 'mountain ecological' regions. Two mountainous districts (Mustang and Humla) were selected for sampling based on past documentation of geography, altitude, population and ethnicity; and the feasibility of conduct for the study. The selected study areas were Jomsom $(2800 \mathrm{~m})$, Jharkot $(3270 \mathrm{~m})$ and Muktinath $(3620 \mathrm{~m})$ of the Mustang district, and Simikot $(2890 \mathrm{~m})$ of the Humla district. In Mustang, the population is predominantly of Tibetan ancestry and that of the Humla district is mainly Indo-Aryan. Both districts are close to the border with China and are heavily influenced by Tibetan culture. Life expectancy at birth is 64.1 years in Mustang and 65.0 years in Humla. Both are lower than the national figure of 68.8 years. $^{6}$

As a group, Tibetans migrated to high mountains of Nepal some 500 years ago. Tibeto-Burman is their native language and they practice Buddhism. Indo-Aryans migrated to Nepal some 2000 years ago, speak Nepalese language and majority of them 
are Hindu. Tibetans and Indo-Aryans are distinctive by appearance, language and name.

Inclusion criteria for participation were age 30 years and above and the ability to speak and understand the Nepalese language. Exclusion criteria were inability or unwillingness to provide written or verbal consent, inability to speak or hear properly and pregnancy.

\section{Sampling process}

Sampling was carried out in two stages. In the first stage, three study areas of Mustang and one study area of Humla were selected on the basis of altitude levels, population density and logistical support to undertake the study. In the second stage, a list of households was developed, and a unique number was assigned to each of them. Household numbers were randomly selected with the help of the computer-based randomisation technique. All eligible family members of the randomly selected households were considered. If none of the household members were eligible or agreed to take part in the study, a household in the close proximity was selected.

The desired sample size within each district was calculated to be around 250 individuals. ${ }^{7}$ This gave a margin of error for a proportion based on anticipated prevalence of HT of around $25 \%$ of plus or minus $5 \%$ after adjusting for the design effect and expected non-response rate of $10 \%$.

\section{Data collection procedures}

The questionnaires administration and BP measurements were carried out at the house of the selected participants. Practically, it was not feasible to undertake blood sample testing, ECG recordings and biophysical measurements in individual houses so a local community hall was used as the measurement facility. Participants were requested to come for these measurements at a time suitable for them. Glycated haemoglobin (HbA1c) and lipid profiles were measured using Cobas b 101 device (Roche Diagnostics). A small amount of capillary whole blood was taken by single finger prick and processed into the device. A 12-lead ECG was recorded using a portable Edan SE-600 instrument. Participants were requested to remove clothing from the chest, lie supine with arms at their sides and legs uncrossed. Oily, sweaty or clammy skin was wiped with alcohol swabs. Qualified female nurses recorded the ECG, however, they were standardised on performing ECGs.

Data were collected during June-August (summer) 2014 in Mustang district and March-May (spring) 2015 in Humla district. The ambient temperature was between $25^{\circ} \mathrm{C}$ and $31^{\circ} \mathrm{C}$ in Mustang and around $15^{\circ} \mathrm{C}$ in Humla on most of study days.

\section{Definitions}

The prevalence of CHD was defined as the presence of pathological Q waves in the ECG (box 1) or self-report of personal

\section{Box 1 Definition of pathological Q waves}

- Any Q wave in leads V2-V3 $\geq 0.02 \mathrm{~s}$ or QS complex in leads $\mathrm{V} 2$ and $\mathrm{V} 3$

- Q-wave $\geq 30 \mathrm{~ms}$ and $\geq 0.1 \mathrm{mV}$ deep or QS complex in leads $\mathrm{I}, \mathrm{II}, \mathrm{aVL}, \mathrm{aVF}$ or V4-V6 in any 2 leads of a contiguous lead grouping (I, aVL, V6; V4-V6; II, III and aVF)

- R-wave $\geq 40$ ms in $\mathrm{V} 1-\mathrm{V} 2$ and $\mathrm{R} / \mathrm{S} \geq 1$ with a concordant positive T-wave in the absence of a conduction defect Thygesen et al. ${ }^{31}$ history of CHD (previous event of myocardial infarction (MI) or chest pain from heart disease (angina)). ECG recordings were categorised as definitely abnormal (eg, showing evidence of previous $\mathrm{MI}$ ), borderline (eg, non-specific T-wave inversion) or normal after review by a cardiologist (SM) using standard widely accepted criteria. The commonly used Sokolow-Lyon index ${ }^{8}$ and Romhilt-Estes score ${ }^{9}$ were used for the diagnosis of left ventricular hypertrophy (LVH). The 10-year risk of fatal or non-fatal cardiovascular events (MI or stroke) was calculated using a validated risk prediction chart designed by WHO and the International Society of Hypertension (ISH) for WHO region of South-East Asia, subregion D. ${ }^{10}$

\section{Statistical methods}

Simple data description is by mean, SD, frequency counts and proportions expressed as percentages. Comparison of age by altitude level was by the Kruskal-Wallis test and $\chi^{2}$ test of independence was used for the subgroup comparisons by sex and ethnicity. The Clopper-Pearson method was used to estimate an exact CI for a single proportion. Logistic regression was used to estimate the association between an abnormal (or borderline abnormal) ECG and altitude. Altitude was treated as a continuous variable in the multivariate model, which also included the variables age, sex, systolic blood pressure (SBP), total cholesterol to high-density lipoprotein-cholesterol ratio, $\mathrm{HbA} 1 \mathrm{c}$ and current smoker. The strength of the associations was considered as strong, moderate and weak at the significance level of $\mathrm{p}<0.01, \mathrm{p}=0.01-0.05$ and $\mathrm{p}=0.06-0.10$, respectively. Stata V.12 was used for data analysis. Analysis was performed for completed ECG recordings only and thus there were no missing points.

This study was approved by the ethical review board of Nepal Health Research Council and the University of Otago, Human Ethics Committee.

\section{RESULTS}

A total population of study area was 2576 , of which study participants were selected from 1587 potential eligible participants aged 30 years or more. The study participants comprised a total of 521 HA residents at three altitude levels: $2800 \mathrm{~m}(\mathrm{~N}=165), 3270 \mathrm{~m}$ $(\mathrm{N}=61)$ and $3620 \mathrm{~m}(\mathrm{~N}=44)$ of the Mustang district and $2890 \mathrm{~m}$ $(\mathrm{N}=251)$ of the Humla district. More than $70 \%$ participants in Mustang and Humla were Tibetans and Indo-Aryans, respectively. Particular ethnic groups among Tibetans were: Thakali, Tibetan Gurung and Lama; and for Indo-Aryans: Brahmin, Chhetri and Dalit. Thirty-six (6.9\%) participants with completed questionnaires and BP measurements did not turn up for ECG recordings and biochemical tests. ECG reports were available for 485 participants. The overall non-response rate was 3.4\%. The most common reason for non-participation was unwillingness to provide blood sample due to the cultural belief of becoming sick. Participants are described in table 1.

More than a quarter of the study participants at the altitude of 2800,3270 and $3620 \mathrm{~m}$ were aged 60 years or more. There was evidence of a difference in age at the different altitude levels; Kruskal-Wallis test $\chi^{2}$ (3 df) 59.1, p <0.01. Women comprised a greater proportion than men at all levels of altitude and overall $55.7 \%$ of study participants were women but there was no evidence this proportion was different by altitude level; $\chi^{2}$ (3 df) $2.5, p=0.47$. Most of the participants were illiterate or had no formal education and reported being born and living at HA for most of their lives. By self-report about half of the participants at all altitude stated they were current consumers of alcohol. 
Table 1 Description of study participants by altitude level

\begin{tabular}{|c|c|c|c|c|c|}
\hline \multirow[b]{3}{*}{ Continuous variables } & & Mustang & & Humla & \multirow[b]{3}{*}{$\mathrm{p}$ Value } \\
\hline & $\begin{array}{l}2800 \mathrm{~m} \\
(\mathrm{~N}=165)\end{array}$ & $\begin{array}{l}3270 \mathrm{~m} \\
(\mathrm{~N}=61)\end{array}$ & $\begin{array}{l}3620 \mathrm{~m} \\
(\mathrm{~N}=44)\end{array}$ & $\begin{array}{l}2890 \mathrm{~m} \\
(\mathrm{~N}=251)\end{array}$ & \\
\hline & \multicolumn{4}{|c|}{ Mean (SD) } & \\
\hline Age & $50.3(13.7)$ & $55.4(13.2)$ & $48.3(12.5)$ & $42.9(11.0)$ & $<0.001$ \\
\hline Average walk time/day (min) & $53.0(44.8)$ & $95.6(79.9)$ & $73.2(53.0)$ & $89.4(89.6)$ & $<0.001$ \\
\hline \multicolumn{6}{|l|}{ Categorical variables } \\
\hline & $\mathrm{N} / 165^{*}(\%)$ & $\mathrm{N} / 61 *(\%)$ & $\mathrm{N} / 44^{*}(\%)$ & $\mathrm{N} / 251^{*}(\%)$ & \\
\hline Sex & & & & & 0.47 \\
\hline Male & $80(48.5)$ & $29(47.5)$ & $19(43.2)$ & $103(41.0)$ & \\
\hline Female & $85(51.5)$ & $32(52.5)$ & $25(56.8)$ & $148(59.0)$ & \\
\hline Education & & & & & $<0.001$ \\
\hline No formal education/illiterate & $67(40.6)$ & $41(67.2)$ & $29(65.9)$ & $168(66.9)$ & \\
\hline Less than primary & $45(27.3)$ & $19(31.1)$ & $6(13.6)$ & $18(7.2)$ & \\
\hline Primary level completed & $25(15.1)$ & $0(0)$ & $4(9.1)$ & $20(8.0)$ & \\
\hline Secondary level completed & $28(17.0)$ & $1(1.6)$ & $5(11.4)$ & $45(17.9)$ & \\
\hline Occupation & & & & & $<0.001$ \\
\hline Government or non-government employee & $25(15.1)$ & $1(1.6)$ & 7 (15.9) & $39(15.5)$ & \\
\hline Self-employed & $86(52.1)$ & $6(9.8)$ & $14(31.8)$ & $21(8.4)$ & \\
\hline Agriculture/daily waged labour & $41(24.8)$ & 39 (63.9) & $21(47.7)$ & $135(53.8)$ & \\
\hline Unemployed/retired/homemaker & $13(7.9)$ & $15(24.6)$ & $2(4.5)$ & $56(22.3)$ & \\
\hline Ethnicity & & & & & $<0.001$ \\
\hline Tibetan & $132(80.0)$ & $58(95.1)$ & $37(84.1)$ & $82(32.7)$ & \\
\hline Indo-Aryans & $33(20.0)$ & $3(4.9)$ & 7 (15.9) & $169(67.3)$ & \\
\hline High-altitude residence & & & & & $<0.001$ \\
\hline For lifetime & $148(89.7)$ & $59(96.7)$ & $38(86.4)$ & $246(98.0)$ & \\
\hline$>10$ years & $17(10.3)$ & $2(3.3)$ & $6(13.6)$ & $2(0.8)$ & \\
\hline $5-10$ years & $0(0)$ & $0(0)$ & $0(0)$ & $3(1.2)$ & \\
\hline Current smoker & $17(10.3)$ & $2(3.3)$ & $4(9.1)$ & $97(38.6)$ & $<0.001$ \\
\hline Current drinker & $84(50.9)$ & 39 (63.9) & $20(45.4)$ & $123(49.0)$ & 0.17 \\
\hline Hypertension or medication & $76(46.1)$ & $25(40.9)$ & $24(54.5)$ & $73(29.1)$ & $<0.001$ \\
\hline Overweight or obesity & $81 / 143(56.6)$ & $16 / 60(26.7)$ & $21(47.7)$ & $52 / 251(20.7)$ & $<0.001$ \\
\hline High TC/HDL ratio or medication & $34 / 139(24.0)$ & $3 / 54(5.6)$ & $11 / 37(29.7)$ & $46 / 167(27.5)$ & $<0.01$ \\
\hline Diabetes or medication & 15/142 (10.6) & 2/61 (3.3) & $6(13.6)$ & 6/172 (3.5) & 0.01 \\
\hline
\end{tabular}

\section{Electrocardiographic analysis \\ Arrhythmias}

Three participants had isolated ectopic beats on their 12-lead ECGs; one atrial, one junctional and one ventricular. Four participants had clear evidence of pre-excitation of the ventricles (Wolff-Parkinson-White syndrome). Four participants showed prolonged atrioventricular (AV) nodal conduction (first-degree AV block) with PR intervals varying between 205 and $286 \mathrm{~ms}$ (normal up to $200 \mathrm{~ms}$ ). One participant had left bundle branch block (LBBB) and six had right bundle branch block (RBBB) (three each complete and incomplete). Two participants, a man and a woman, had prolonged QT intervals; 470 and $490 \mathrm{~ms}$.

\section{Morphological abnormalities}

Two participants had tall peaked $\mathrm{P}$ waves suggesting right atrial hypertrophy. Three participants showed extreme axis deviation (arm lead electrode positions all verified as correct) with QRS axes between $+185^{\circ}$ and $+237^{\circ}$ suggesting possible congenital abnormalities and/or pulmonary HT. Twenty-six other participants showed right axis deviation (RAD), with QRS axes between $92^{\circ}$ and $143^{\circ}$, of which two had right ventricular hypertrophy (RVH). Four others had left axis deviation (LAD), with QRS axes between $-34^{\circ}$ and $-85^{\circ}$. No participant had Q waves to suggest past Q-wave MI. Thirteen had inverted T waves outside the accepted normal leads (III, aVR, V1), nine of these were of doubtful significance as they were in the anteroseptal precordial leads V1-V4. Two had inverted T waves in inferior leads (II, III and aVF) and two in aVL alone. Seventeen participants met Sokolow-Lyon criteria for $\mathrm{LVH}$, with the largest $\mathrm{S}$ in $\mathrm{V} 1$ or $\mathrm{V} 2$ plus the largest $\mathrm{R}$ in V5 or V6 $>3.5 \mathrm{mV}$, but only one of these met the stricter definition of Romhilt and Estes (six points), three others were borderline (four points). Two additional participants had down-sloping ST segments with no other cause but suggestive of hypertrophy.

Overall, none of the participants showed a definite electrocardiographic evidence of CHD. Overall, 27/485, 5.6\% (95\% CI 3.7 to 8.0 ), of the participants gave a self-report of a history of CHD confirmed by a medical doctor. The prevalence of CHD was $17 / 212(8.0 \%)$ in men and $10 / 273(3.7 \%)$ in women, $\chi^{2}(1$ df) $4.3, \mathrm{p}=0.04$. Altogether, 95/485, 19.6\% (95\% CI 16.1 to 23.4), of the participants had an abnormal (or borderline abnormal) ECG. The main abnormalities were: RAD in $26 / 485,5.4 \%$ (95\% CI 3.5 to 7.7 ) and LVH by voltage criteria in $17 / 485$, $3.5 \%(95 \%$ CI 2 to 5.5$)$. Of 26 participants with RAD, 
$16(61.5 \%)$ were men and $13(50 \%)$ were from the age group 30-39 years. Among 17 participants with LVH, 11 (64.7\%) were women, $12(70.6 \%)$ were aged 50 years or more and $11(70.6 \%)$ were hypertensive or on antihypertensive treatment.

Participants resident living in the Mustang district at altitudes of 2800,3270 and $3620 \mathrm{~m}$ mostly had abnormalities on the left side of the heart. For Humla participants at $2890 \mathrm{~m}$, the main reasons for the abnormality were mostly on the right side. For example, Mustang participants had 15/243 (6.2\%) ECG findings of LVH, LAD or LBBB compared with $7 / 242$ (2.9\%) Humla participants: $\chi^{2}$ ( $1 \mathrm{df}$ ) $3.01, \mathrm{p}=0.08$. In Humla, 21/242 $(8.7 \%)$ ECG reports had RAD, RBBB or right atrial hypertrophy compared with $13 / 243(5.3 \%)$ in the Mustang participants: $\chi^{2}(1 \mathrm{df}) 2.06, \mathrm{p}=0.15$

Figure 1 shows the proportions of participants with abnormal (or borderline abnormal) ECG and with WHO/ISH 10-year risk of fatal or non-fatal cardiovascular events (MI or stroke) of $>10 \%$ by altitude level. More than one-fourth of the participants at $3270 \mathrm{~m} \mathrm{(30 \% )} \mathrm{and} 3620 \mathrm{~m} \mathrm{(29.5 \% )} \mathrm{had} \mathrm{an} \mathrm{abnormal}$ (or borderline abnormal) ECG, nearly twofold higher than those living at $2800 \mathrm{~m} \mathrm{(15.8 \% )} \mathrm{and} 2890 \mathrm{~m}$ (17.4\%). Residents at higher altitudes of 3270 and $3620 \mathrm{~m}$ were also more likely to have a predicted $>10 \%$ risk of a fatal or non-fatal major cardiovascular event over the next 10 years.

There was a moderate association between the probability of an abnormal (or borderline abnormal) ECG and altitude when adjusted for potential confounding variables in a multivariate logistic model (table 2). There were 99 missing data points in multivariate model and there were no statistically significant differences between ECG reports (abnormal or normal) and data status (missing or not missing).

\section{DISCUSSION}

The prevalence of CHD in this HA population is estimated to be $5.6 \%$. This is based on personal history alone as no participant had ECG evidence of past Q-wave MI. The prevalence was higher in men. The prevalence of an abnormal (or borderline abnormal) ECG was between $16 \%$ and $30 \%$ in different HA areas sampled for this study from Nepal. After adjusting for the important confounding variables, the odds of having an abnormal (or borderline abnormal) ECG increased nearly threefold for every $1000 \mathrm{~m}$ increment in altitude. This association was particularly strong for men. RAD and LVH were the most common abnormalities.

Our result is strikingly similar to available evidence derived from ECG recordings at low altitude of Nepal, China and Asia;

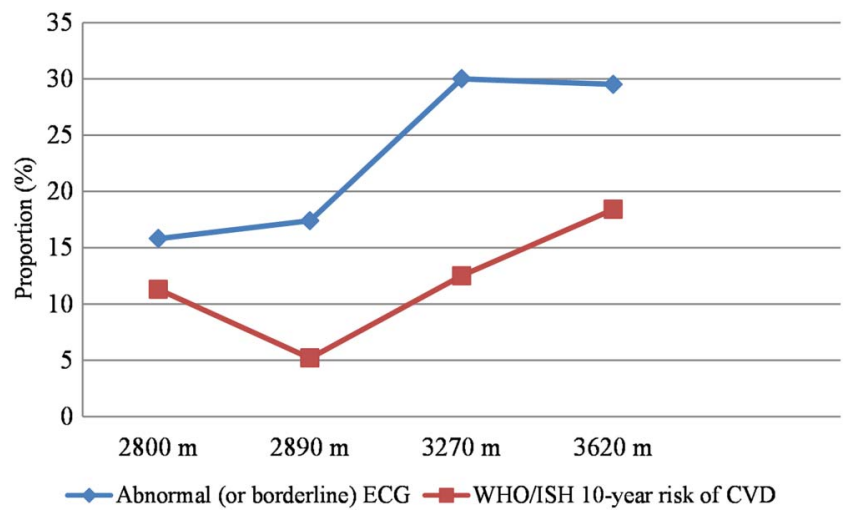

Figure 1 Proportions of abnormal (or borderline) ECG and WHO/ International Society of Hypertension 10-year risk of fatal or non-fatal cardiovascular disease (CVD) events by $>10 \%$ at each altitude level. and close to that of rural areas of South Asian countries. Electrocardiographic evidence for CHD at the population level is sparse in Nepal to allow comparisons for our study results. Based on ECG and documented medical history, only one population-based sample survey has been reported at low altitude of Nepal and among men only documented the prevalence of $\mathrm{CHD}$ at $5.7 \% .^{11} \mathrm{~A}$ systematic review of global studies of CHD prevalence based on ECG reports or documented medical history in adults and including publications through to 2014 estimated the prevalence of CHD in Asia at 5.8\%, between $5.6 \%$ and $5.9 \%$ in rural China, between $1.4 \%$ and $4.6 \%$ in rural areas of India, 3.4\% in rural area of Bangladesh and 5.1\% in urban Pakistan. ${ }^{12}$ Although there have been few populationbased studies on CHD in HA populations living at $2500 \mathrm{~m}$ or more worldwide, our findings are consistent with the majority of hospital-based reports ${ }^{13}{ }^{14}$ but in contrast to few others. ${ }^{15} 16$ The most recent systematic analysis of the burden of disease in Chinese provinces showed that the age-standardised mortality rate from CHD in Tibet (average altitude of $4500 \mathrm{~m}$ from sea level) was significantly lower in men but similar in women when compared with those living at lower altitudes. ${ }^{17}$ The prevalence of ECG abnormalities in this study was also lower when compared with other ECG-based studies in HA natives. ${ }^{18-20}$ It should be noted that studies reporting markedly higher prevalence were conducted in higher levels of altitude compared with our study and this may partly explain the differences.

A review of the English language literature on ECG recordings at different levels of altitude above $2500 \mathrm{~m}$ identified no studies. Thus, our finding of significant association between abnormal (or borderline) ECG and altitude is difficult to compare with other studies. However, one study did report increasing rates of RAD with increasing levels of altitude in HA setting. ${ }^{21}$ In our study, there was a positive relationship between SBP and altitude in a multivariate model adjusting for potential confounders. This relationship may explain the increased prevalence of an abnormal (or borderline) ECG with increasing levels of altitude. For example, more than two-thirds of the participants with LVH were hypertensive or on treatment. There are important differences in the prevalence of overweight or obesity, diabetes and walking time by altitude level, which may be attributed to urban $(2800$ and $3620 \mathrm{~m}$ ) and rural $(2890$ and $3270 \mathrm{~m})$ residential settings. The main reason behind low prevalence of current smoker in Tibetan dominant study areas is cultural non-preference to tobacco smoking.

The low prevalence of CHD in the study reported here may have several possible explanations. The reasons proposed for possible cardioprotection in HA residents includes activation of a transcriptor-modulator hypoxia-inducible factor 1, which

Table 2 Estimates of the OR for abnormal or borderline ECG by altitude in a multivariate logistic model and associations for confounding variables

\begin{tabular}{lll}
\hline Variables and comparison & OR (95\% Cl) & $\mathbf{p}$ Value \\
\hline Altitude (per $1000 \mathrm{~m}$ higher) & $2.83(1.07$ to 7.45$)$ & 0.03 \\
Systolic blood pressure (per $10 \mathrm{~mm} \mathrm{Hg}$ higher) & $1.25(1.08$ to 1.44$)$ & 0.002 \\
TC-to-HDL ratio (per unit ratio higher) & $0.93(0.75$ to 1.15$)$ & 0.49 \\
HbA1c (per mmol/mol higher) & $1.02(0.99$ to 1.05$)$ & 0.49 \\
Smoke tobacco (yes compared with no) & $1.69(0.97$ to 2.94$)$ & 0.06 \\
Age (per decade older) & $1.14(0.91$ to 1.43$)$ & 0.24 \\
Sex (men compared with women) & $1.69(0.97$ to 2.94$)$ & 0.06 \\
\hline HbA1c, glycated haemoglobin; HDL, high-density lipoprotein-cholesterol; TC, total \\
cholesterol.
\end{tabular}


induces the expression of multiple genes with cardioprotective properties. ${ }^{22}$ High levels of vitamin $\mathrm{D}$ due to an exponential increase in ultraviolet $\mathrm{B}$ light at $\mathrm{HA},{ }^{23}$ improvement in coronary vasculature through increased number of branches and peripheral vessels, ${ }^{24}$ increased levels of physical fitness and low level of air pollution ${ }^{25}$ could also provide cardioprotection. A 'healthy individuals effect' is an alternative explanation to these findings from non-experimental studies of illness at HA because sick people often migrate to LA for better treatment. ${ }^{26}$

It is plausible that the CHD-related risk pattern at $\mathrm{HA}$ is also ethnicity specific due to varying degree of adaptation, physiological response, lifestyle and genetic factors. Contrasting cardiopulmonary responses to HA exposure among the largest HA populations, Tibetans, Andeans and Aymaras, have been documented. ${ }^{27}$ The finding in this study that abnormal or borderline abnormal ECG recordings on the left side of the heart were more prevalent among those of the Tibetan ethnicity (Mustang district), whereas right-sided abnormalities were more frequent in Humla district with majority of Indo-Aryan participants, may reflect this phenomena. The ECG abnormalities suggesting right heart abnormalities may reflect increased pulmonary vascular resistance; well reported at greater level among HA dwellers less adapted with hypoxia such as Andeans or North Americans, ${ }^{19} 28$ but at lower level in those with high degree of adaptation such as Tibetans. ${ }^{29}$ The high prevalence of RAD in our study, particularly in Humla participants, also suggests a possible early indication of pulmonary HT or RVH. A previous report suggested RAD could be up to four times more prevalent at HA compared with sea level presumably correlating with differences in pulmonary artery pressure. ${ }^{30}$

As far as we are aware, this is the only study which has provided detailed information on CHD health and comprehensively assessed cardiovascular risk among HA natives in Nepal. Limitations of this study included possible bias due to the purposive selection of the study areas on the basis of altitude levels, population density and logistical convenience. Validity of the self-report of CHD is also questionable particularly due to the poor literacy rate of the participants. Reported prevalence might not reflect true incidence due to the 'healthy individual effect'. Difference in temperature in Mustang and Humla districts during data collection

\section{Key messages}

What is already known about this subject?

The balance of risks and benefits for cardiovascular health in permanent residents of high altitude is uncertain. Different patterns of adaptation and physiological responses against hypobaric hypoxia as well as different lifestyle practices of high-altitude populations probably contribute.

What does this study add?

This is first ECG-based study examining evidence of coronary heart disease in high-altitude populations of Nepal. The prevalence of coronary heart disease in high-altitude populations may be comparable with those from low-altitude residents. However, ECG abnormalities may increase by altitude and the risk pattern may vary by ethnicity; mostly on the right side of the heart in Indo-Aryans and on the left side in Tibetans.

How might this impact on clinical practice? Clinicians should be aware of differential effects of high-altitude living on cardiovascular health by ethnicity. period may confound observed association. There were possible confounding variables which are commonly relevant for CHD. There might be other unmeasured confounding variables particularly related to the HA populations.

In conclusion, our findings based on personal history and ECG recordings suggest that HA populations of this study area had a prevalence of CHD comparable with that seen in low-altitude populations. However, we demonstrated moderate evidence of an association between participants having an abnormal (or borderline abnormal) ECG and altitude in our HA populations in Nepal. The risk pattern may vary by ethnicity; increased pulmonary vasoconstriction reflected by RAD in Indo-Aryans (Humla district) and HT-induced LVH in participants of Mustang with Tibetan ancestry. Clinicians should be aware of differential effects of HA living on cardiovascular health by ethnicity. More useful epidemiology may depend on better information infrastructure in the relevant population (eg, improvement in registration of births, deaths and marriages). Directly comparable data compilation from residents living at low altitude and HA would add clarity to attribution of health status to altitude per se.

Acknowledgements We would like to specially thank research assistants Prakash Pant, Ashish Khadayat and Mamata Chand for their incredible support in data collection. We are grateful to local nurses Sofia Shrestha, Reena Thakali and female community health volunteers Lalita Sherchan (Mustang) and Guyala Rawat (Humla), who helped significantly in various aspects of data collection.

Contributors All authors contributed to study design. NA collected the data, performed statistical analysis and prepared the first draft of the manuscript. YKDB helped with data collection and MW helped with the statistical analysis. SM reviewed and interpreted the ECG reports. All authors contributed intellectually to the revision of the article and approved the final version.

Funding Capital Cardiovascular Research Trust, Wellington, New Zealand provided funding for the study as well as provided financial support for PhD study of NA at the University of Otago, Wellington, New Zealand.

Competing interests None declared.

Ethics approval University of Otago, Human Ethics Committee; Nepal Health Research Council.

Provenance and peer review Not commissioned; externally peer reviewed.

\section{REFERENCES}

1 Moore L G, Niermeyer S, Zamudio S. Human adaptation to high altitude: regional and life-cycle perspectives. Am J Phys Anthropol 1998;107(Suppl 27):25-64.

2 Ostadal B, Kolar F. Cardiac adaptation to chronic high-altitude hypoxia: beneficial and adverse effects. Respir Physiol Neurobiol 2007;158:224-36.

3 Beall CM. Two routes to functional adaptation: Tibetan and Andean high-altitude natives. Proc Natl Acad Sci USA 2007;104(Suppl 1):8655-60.

4 Yi X, Liang Y, Huerta-Sanchez E, et al. Sequencing of 50 human exomes reveals adaptation to high altitude. Science 2010;329:75-8

5 Central Bureau of Statistics. Nepal in figures. Kathmandu, Nepal: Government of Nepal, National Planning Commission Secretariat, 2012.

6 Government of Nepal \& United Nations Development Programme. Nepal human development report 2014: beyond geography, unlocking human potential. In: Luchsinger G, ed. Kathmandu, Nepal, 2014:90-92.

7 Daniel WW. Biostatistics: a foundation for analysis in the health sciences. 7th edn. New York: John Wiley \& Sons, 1999.

8 Sokolow M, Lyon TP. The ventricular complex in left ventricular hypertrophy as obtained by unipolar precordial and limb leads. Am Heart J 1949;37:161-86

9 Romhilt DW, Estes EH. A point-score system for the ECG diagnosis of left ventricular hypertrophy. Am Heart J 1968;75:752-8.

10 Mendis S, Lindholm LH, Mancia G, et al. World Health Organization (WHO) and International Society of Hypertension (ISH) risk prediction charts: assessment of cardiovascular risk for prevention and control of cardiovascular disease in low and middle-income countries. J Hypertens 2007;25:1578-82.

11 Vaidya A, Pokharel PK, Nagesh S, et al. Prevalence of coronary heart disease in the urban adult males of eastern Nepal: a population-based analytical cross-sectional study. Indian Heart J 2009;61:341-7.

12 Zhu KF, Wang YM, Zhu JZ, et al. National prevalence of coronary heart disease and its relationship with human development index: a systematic review. Eur J Prev Cardiol 2016;23:530-43.

13 Hurtado A. Some clinical aspects of life at high altitudes. Ann Intern Med 1960;53:247-58 
14 Ramos A, Krüger H, Muro M, et al. Pathology of natives of high altitudes. Study of the causes of death in 300 autopsies. Boletin de la Oficina Sanitaria Panamericana Pan American Sanitary Bureau 1967;62:496-502.

15 Zhao D. [The epidemiology of coronary heart disease (CHD) in 16 provinces of China. Multi-province Cooperative Group of Cardiovascular Disease Surveillance (MONICA Project)]. Zhonghua liu xing bing xue za zhi 1993;14: 10-13.

16 Al-Huthi MA, Raja'a YA, Al-Noami M, et al. Prevalence of coronary risk factors clinical presentation, and complications in acute coronary syndrome patients living at high vs low altitudes in Yemen. Med Gen Med 2006;8:28.

17 Zhou M, Wang H, Zhu J, et al. Cause-specific mortality for 240 causes in China during 1990-2013: a systematic subnational analysis for the Global Burden of Disease Study 2013. Lancet 2016;387:251-72.

18 Halperin BD, Sun S, Zhuang J, et al. ECG observations in Tibetan and Han residents of Lhasa. J Electrocardiol 1998;31:237-43.

19 Rotta A, Lopez A. Electrocardiographic patterns in man at high altitudes. Circulation 1959;19:719-28.

20 Kaushal SS, DasGupta DJ, Prashar BS, et al. Electrocardiographic manifestations of healthy residents of a tribal Himalayan village. J Assoc Physicians India 1995:43:15-16.

21 Raynaud J, Valeix P, Drouet L, et al. Electrocardiographic observations in high altitude residents of Nepal and Bolivia. Int J Biometeorol 1981;25:205-17.
22 Semenza GL. Regulation of physiological responses to continuous and intermittent hypoxia by hypoxia-inducible factor 1. Exp Physiol 2006;91:803-6.

23 Anderson JD, Honigman B. The effect of altitude-induced hypoxia on heart disease: do acute, intermittent, and chronic exposures provide cardioprotection? High Alt Med Biol 2011;12:45-55.

24 Anatomy of the coronary circulation at high altitude. Ciba foundation symposium-high altitude physiology: cardiac and respiratory aspects. Wiley Online Library, 1971.

25 Burtscher M. Effects of living at higher altitudes on mortality: a narrative review. Aging Dis 2014;5:274.

26 Regensteiner JG, Moore LG. Migration of the elderly from high altitudes in Colorado. JAMA 1985;253:3124-8.

27 Hurtado A, Escudero E, Pando J, et al. Cardiovascular and renal effects of chronic exposure to high altitude. Nephrol Dial Transplant 2012;27(Suppl 4):iv11-16.

28 Pryor R, Weaver WF, Blount SG. Electrocardiographic observation of 493 residents living at high altitude (10,150 feet). Am J Cardiol 1965;16:494-9.

29 Groves BM, Droma T, Sutton JR, et al. Minimal hypoxic pulmonary hypertension in normal Tibetans at 3,658 m. J Appl Physiol 1993;74:312-8.

30 Windsor JS, Rodway GW, Montgomery HE. A review of electrocardiography in the high altitude environment. High Alt Med Biol 2010;11:51-60. http://dx.doi.org/ 10.1089/ham.2009.1065

31 Thygesen K, Alpert JS, Jaffe AS, et al. Third universal definition of myocardial infarction. Circulation 2012;126(16):2020-35. 